\title{
New Sphingolipids from Marine Sponge Iotrochota baculifera
}

\author{
Pendyala Muralidhar, ${ }^{a}$ Nallamothu Krishna, ${ }^{a}$ Muthyala Muralikrishna Kumar, ${ }^{a}$ \\ Chaganty Bheemasankara RAo, ${ }^{b, 1)}$ and Desaraju Venkata RAO ${ }^{* a}$ \\ ${ }^{a}$ Department of Pharmaceutical Sciences, Andhra University; and ${ }^{b}$ Department of Organic Chemistry, Andhra University; \\ Visakhapatnam-530003, India. Received May 29, 2003; accepted July 23, 2003
}

\begin{abstract}
Chemical examination of the marine sponge Iotrochota baculifera of the Indian Ocean furnished a sphingolipid (1), a new glycosphingolipid (iotroridoside-B, 2) and a four-component mixture of sphingolipids (3a-d) with two new components. Structure elucidation was carried out by spectral and chemical methods.
\end{abstract}

Key words Iotrochota baculifera; sphingolipids; iotroridoside-B; marine sponge; Myxillidae

Sphingolipids which are present widely in the membranes of eukaryotic cells of both terrestrial and marine organisms involve in many a physiological process and a few exhibited biological activities like cytotoxic, anti-inflammatory etc. as well. $^{2-4)}$ We report in this communication the isolation and characterization of a sphingolipid (1), a glycosphingolipid (2) and a mixture of sphingolipid homologues (3a- d) from the marine sponge Iotrochota baculifera (Fam: Myxillidae) of the Indian Ocean. Earlier records on this genus include alkaloids, a toxin, ecdysteroids, sphingolipids, bromoindole and tyrosine derivatives. ${ }^{3,4)}$

\section{Results and Discussion}

Separation of EtOAc extract by medium pressure liquid chromatograph (MPLC) yielded compounds $\mathbf{1}, \mathbf{2}$ and $\mathbf{3 a -}-\mathbf{d}$ as colourless crystals and showing the presence of hydroxyl and amide $\mathrm{NH}\left(3500-3200 \mathrm{~cm}^{-1}\right)$ and amide carbonyl $\left(1640 \mathrm{~cm}^{-1}\right)$ in the IR spectra.

Compound $1, \mathrm{mp} 145^{\circ} \mathrm{C}$, was analyzed for $\mathrm{C}_{43} \mathrm{H}_{87} \mathrm{NO}_{5}$ by the fast atom bombardment mass spectrometry (FAB-MS) and elemental analysis. The ${ }^{13} \mathrm{C}-\mathrm{NMR}$ spectrum has signals appeared due to amide carbonyl at $\delta 175.3$ and a methine carbon at $\delta$ 53.0. The ${ }^{1} \mathrm{H}-\mathrm{NMR}$ spectrum showed signals of an amide proton doublet at $\delta 8.58(1 \mathrm{H}, J=8.7 \mathrm{~Hz})$ and protons of a long methylene chain at $\delta 1.29$ (brs) indicating a sphingolipid skeleton. ${ }^{5,6)}$ The absence of singlets at $\delta 1.90$ 2.25 in ${ }^{1} \mathrm{H}-\mathrm{NMR}$ spectra suggesting the absence of acetoxyls in 1. Due to overlapping of signals in NMR spectra, $\mathbf{1}$ was acetylated and spectra recorded for acetate $1 \mathrm{a}$. The ${ }^{1} \mathrm{H}$ - and ${ }^{13} \mathrm{C}$-NMR spectra showed four acetoxy methyl signals at $\delta$ $2.03-2.18(12 \mathrm{H}, \mathrm{s})$ and $20.6-21.0$, which revealed the presence of four hydroxyls in 1 . The absence of a proton triplet at $\delta 2.22-2.50$ in ${ }^{1} \mathrm{H}-\mathrm{NMR}$ spectrum suggested the presence of $-\mathrm{CO}-\mathrm{CH}(\mathrm{OAc})-\mathrm{CH}_{2}-$ moiety in $1 \mathbf{a}^{6,7)}$ The positions of oxygenated carbons in $1 \mathbf{a}$ were assigned on the basis of ${ }^{1} \mathrm{H}-{ }^{1} \mathrm{H}$ correlation spectroscopy (COSY) and heteronuclear multiple quantum coherence (HMQC) spectra which led to the proposal that $\mathbf{1}$ is a sphingolipid containing 1,3,4trihydroxy base and a 2-hydroxy fatty acid and without unsaturation in fatty chains.

The length of the fatty acid and sphingosine chains in $\mathbf{1}$ were determined by methanolysis of $\mathbf{1}$ followed by acetylation of sphingamines according to method of Gaver and Sweeley. ${ }^{8)}$ The methyl ester and tetraacetylated sphingamines thus obtained were independently analysed by gas chromatography-mass spectrometry (GC-MS), which showed ions at $\mathrm{m} / \mathrm{z} 412$ and 485 corresponding to methyl 2-hydroxy pentacosanoate and 1,3,4-triacetyl-2-acetamino-octadecane respectively. Based on the NMR and optical rotation values of natural and synthetic methyl esters and sphingamines, ${ }^{5,9)} R$ and $2 S, 3 S, 4 R$ configurations were proposed to methyl ester and sphingamine moieties respectively. The structure of $\mathbf{1}$ could be described as $(2 S, 3 S, 4 R)-2-[(2 R)$-2-hydroxy pentacosanoylamino]-octadeca-1,3,4-triol. Literature has two reports of occurrence of this sphingolipid from non-marine sources, mushrooms Phellinus ribis ${ }^{10)}$ and Grifola frondosa. ${ }^{11)}$

Compound 2, mp $205^{\circ} \mathrm{C}$, was analyzed for $\mathrm{C}_{48} \mathrm{H}_{93} \mathrm{NO}_{10}$ from the positive FAB-MS and elemental analysis. The presence of an amide $\mathrm{NH}$ at $\delta 8.61(\mathrm{~d}, J=9 \mathrm{~Hz})$, a long chain of methylene protons at $\delta 1.29$ (br s), two terminal methyls at $\delta$ $0.85(\mathrm{t}, J=6.9 \mathrm{~Hz})$, an anomeric proton at $\delta 4.94(\mathrm{~d}, J=8 \mathrm{~Hz})$ and protons on oxygenated carbons appearing as multiplets between $\delta 3.86$ and 4.75 in ${ }^{1} \mathrm{H}-\mathrm{NMR}$ of $\mathbf{2}$ suggested it to be a

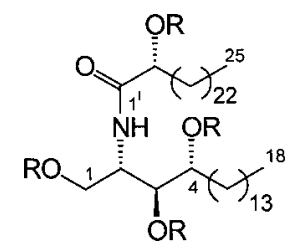

$\mathbf{1}, \mathrm{R}=\mathrm{H} ; \mathbf{1} \mathbf{a}, \mathrm{R}=\mathrm{Ac}$

Fig. 1

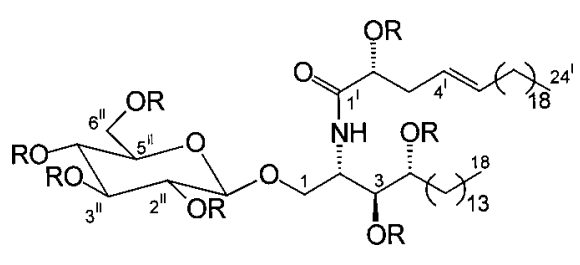

2, $\mathrm{R}=\mathrm{H} ; \mathbf{2} \mathbf{a}, \mathrm{R}=\mathrm{Ac}$

Fig. 2

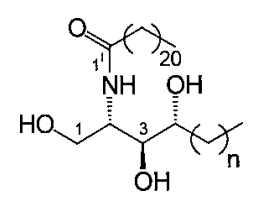

3a, $n=14 ; \mathbf{3 b}, n=15 ; \mathbf{3 c}, n=16 ; \mathbf{3 d}, n=17$

Fig. 3 
glycosphingolipid. ${ }^{5)}$ The appearance of carbon resonances at $\delta 62.6,71.5,75.2,78.5,78.6$ and 105.6 and a correlation between $\delta 4.94$ and 105.6 in HMQC spectrum are attributable to $\beta$-glucopyranose as sugar moiety in $\mathbf{2}$. $^{5,12}$

The presence of seven acetoxy methyl groups resonating between $\delta 1.99$ and 2.24 in ${ }^{1} \mathrm{H}-\mathrm{NMR}$ spectrum of peracetylated derivative of $\mathbf{2}$ indicates the presence of three hydroxyls in the sphingolipid skeleton, in addition to four on glucopyranose unit of $\mathbf{2}$. The positions of hydroxyl groups in the ceramide part were ascertained by ${ }^{1} \mathrm{H}-{ }^{1} \mathrm{H}$ COSY data which assigned two double doublets at $\delta 4.75$ and 4.55 and two multiplets at $\delta 4.34$ and 4.20 to protons $\mathrm{H}-1 \mathrm{a}, \mathrm{H}-1 \mathrm{~b}, \mathrm{H}-3$ and $\mathrm{H}-4$ respectively. The ${ }^{1} \mathrm{H}-{ }^{1} \mathrm{H}$ COSY spectrum also showed the presence of a double bond and HMQC spectrum showed correlation between olefinic carbons at $\delta 132.4$ and 126.3 with protons at $\delta 5.65(1 \mathrm{H}, \mathrm{dt}, J=7.2,17.7 \mathrm{~Hz})$ and $5.96(1 \mathrm{H}, \mathrm{dt}$, $J=7.2,18.0 \mathrm{~Hz})$ respectively. The olefinic proton at $\delta 5.96$ showed correlation for two methylene protons each at $\delta 2.88$ and 3.05. These two methylene protons are inter-correlated and correlated to a proton on the hydroxylated carbon appearing at $\delta 4.64$ thus indicating the presence of $-\mathrm{CO}-$ $\mathrm{CH}(\mathrm{OH})-\mathrm{CH}_{2}-\mathrm{CH}=\mathrm{CH}-$ moiety which was further evidenced by the absence of a triplet at $\delta 2.25-2.50 .{ }^{6,7)}$ From the observations it may be summerised that a methylene intervenes between the hydroxylated carbon and the olefinic carbon in fatty acyl chain. The other olefinic proton at $\delta 5.65$ $\left(\mathrm{H}-5^{\prime}\right)$ showed cross peaks with methylene protons at $\delta 2.16$, which further showed cross peaks with long-chain methylene protons at $\delta 1.29$. The coupling constant between the olefinic protons $(17.7 \mathrm{~Hz})$ and chemical shifts of the adjacent carbons at $\delta 33.6$ and 32.2 strongly suggest trans $(E)$ orientation (a cissoid orientation is expected to have a $J$ value of $6-12 \mathrm{~Hz}$ and adjacent carbon shifts at $\cong 27 \mathrm{ppm}) .{ }^{5,13,14)}$ All the assignments were further confirmed by HMQC spectra.

The fatty acid and sphingosine chain lengths and sugar of 2 were determined through methanolysis according to Gaver and Sweeley. ${ }^{8}{ }^{1} \mathrm{H}-\mathrm{NMR}$ of the ester so obtained on methanolysis showed the presence of olefinic protons at $\delta 5.34$ $(1 \mathrm{H}, \mathrm{dt}, J=17.7,7.2 \mathrm{~Hz}, \mathrm{H}-5)$ and $5.54(1 \mathrm{H}, \mathrm{dt}, J=7.2$, $17.4 \mathrm{~Hz}, \mathrm{H}-4)$. GC-MS analysis showed the presence of the methyl 2-hydroxy 4-tetracosenoate $(\mathrm{m} / \mathrm{z} 396)$. On comparison with the literature data, ${ }^{5)}$ the optical rotation $[\alpha]_{\mathrm{D}}^{28}-2.4^{\circ}$ indicated $R$ configuration for the ester. The sphingosine base and methyl $\beta$-glucoside were separated from aqueous mathanolic layer. The sphingosine base, after acetylation followed by chromatography over Sephadex LH20, gave the tetraacetyl sphingamine moiety which on GC-MS analysis showed one ion at $\mathrm{m} / \mathrm{z} 485$ for $\left[\mathrm{M}^{+}\right]$and $\mathrm{m} / \mathrm{z} 426$ for $[\mathrm{M}-\mathrm{AcO}]^{+}$, corresponding to the $1,3,4$-triacetoxy-acetamino octadecane. On the basis of NMR and optical rotation data in the literature, ${ }^{5,9)} 2 S, 3 S, 4 R$ configuration could be proposed for the sphingamine base. The sugar residue obtained from the aqueous layer was identified as methyl- $\beta$-D-glucopyranoside by comparing the spectral data and optical rotation with those reported for authentic sample. ${ }^{5)}$

From the foregoing discussion, compound $\mathbf{2}$ could be characterized as $(2 S, 3 S, 4 R)-1-O$ - $(\beta$-D-glucopyranosyl)-2$[(2 R, 4 E)$-2-hydroxy-4-tetracosenoylamino]-octadecane-1,3,4triol. As literature survey indicated that the compound iotroridoside- $\mathrm{A}^{15)}$ has the same structure excepting ' $Z$ ' configuration at the double bond, $\mathbf{2}$ was named as iotroridoside-B.
The FAB-MS of $\mathbf{3}$ showed a series of pseudomolecular ion peaks $[\mathrm{M}+\mathrm{H}]^{+}$at $m / z 654,668,682$ and 696 suggesting a mixture of homologues. As attempts to separate the constituents by HPLC including reverse phase were not successful, the spectra were recorded for the mixture. A few differences were noticed by a scrutiny of spectral data of $\mathbf{1}$ and $\mathbf{3}$ viz. the presence of a triplet at $\delta 2.45$ and three acetoxy methyls in ${ }^{1} \mathrm{H}-\mathrm{NMR}$ spectrum of $\mathbf{3}$ and its acetate respectively, showing three hydroxyls and a non-hydroxy fatty acid in 3. The positions of hydroxyls in $\mathbf{3}$ were assigned on the basis of ${ }^{1} \mathrm{H}-{ }^{1} \mathrm{H}$ COSY data (see experimental section).

The length of the fatty acid and sphingosine chains in $\mathbf{3}$ were determined by methanolysis ${ }^{8)}$ which gave methyl ester and sphingamines. The GC-MS of the methyl ester showed an ion at $\mathrm{m} / \mathrm{z} 354$ corresponding to methyl docosanoate wheras the acetylated sphingamines showed four ions at $\mathrm{m} / \mathrm{z}$ $499(23 \%), 513(26 \%), 527(23 \%)$ and 541 (28\%) corresponding to 1,3,4-triacetyl-2-acetamino-substituted nonadecane, eicosane, heneicosane and docosane respectively. From the foregoing discussion it may be proposed that $\mathbf{3}$ is a mixture of sphingolipids containing 1,3,4-trihydroxy-2-docosanoylamino-nonadecane (3a), 1,3,4-trihydroxy-2-docosanoylamino-eicosane (3b), 1,3,4-trihydroxy-2-docosanoylamino-heneicosane (3c) and 1,3,4-trihydroxy-2-docosanoylamino-docosane (3d). Of this $\mathbf{3 a}$ and $\mathbf{3 c}$ are new to the literature while $\mathbf{3 b}$ and $\mathbf{3 d}$ have been reported earlier. ${ }^{16,17)}$

\section{Experimental}

General ${ }^{1} \mathrm{H}$ - and ${ }^{13} \mathrm{C}$-NMR spectra were recorded on a Bruker DRX spectrometer operating at $300 \mathrm{MHz}$ and $75 \mathrm{MHz}$ respectively. The chemical shift values were reported in parts per million units and the coupling constants were in Hz. FAB-MS was recorded on a JEOL-Sx-120/DA-6000 mass spectrometer using a beam of Argon/Xenon ( $2-8 \mathrm{keV})$ and m-nitrobenzyl alcohol as the matrix. Optical rotations were taken on a JASCO DIP-370 polarimeter. Elemental analysis was carried out on a Carlo Erba 1108 analyzer. IR spectra were recorded on a Perkin-Elmer 881 instrument. Melting points were recorded on Boitus melting point apparatus and were uncorrected. $\mathrm{Si}$ gel column chromatography was carried out using silica gel (finer than 200\#, ACME), gel filtration was carried out using LH20 (Sephadex LH20, Pharmacia Biotech) and MPLC was performed on Buchi B-688 MPLC system. GC-MS experiments were carried out on Shimadzu's GCMS-QP 5050A system. HPLC was carried out on Shimadzu HPLC class VP series with two LC-10AT VP pumps and UV/Vis Detector SPD-10A VP.

Animal Material Specimens of the sponge Iotrochota baculifera were collected from the Coast near Tuticorin, India $\left(8^{\circ} 45^{\prime} \mathrm{N}, 78^{\circ} 12^{\prime} \mathrm{E}\right)$ at a depth of $10-15 \mathrm{~m}$ and preserved in methanol until extraction. A voucher specimen was deposited in the Marine organisms museum in the Department of Pharmaceutical Sciences, Andhra University, Visakhapatnam, India (Voucher No. AU2-152).

Extraction and Isolation The organism was extracted with $\mathrm{MeOH}$ eight times at room temperature on every $48 \mathrm{~h}$. The combined alcoholic extract was concentrated under reduced pressure. The concentrated extract was fractionated with EtOAc and the EtOAc fractions were pooled and concentrated under reduced pressure after drying on anhydrous $\mathrm{MgSO}_{4}$ to yield a crude residue $(21 \mathrm{~g})$. The residue $(18 \mathrm{~g})$ was column chromatographed over silica gel ( $400 \mathrm{~g}$, finer than 200\#, ACME, column diameter $60 \mathrm{~mm}$ ) using eluants with increasing polarity starting from $n$-hexane through EtOAc to $\mathrm{MeOH}$. The fractions eluted with $40 \%$ and $60 \%$ EtOAc in hexane and EtOAc were pooled and concentrated to yield 3a-d (40 mg), $1(120 \mathrm{mg})$ and $\mathbf{2}(28 \mathrm{mg})$ respectively. The mixture $\mathbf{3 a}-\mathbf{d}$ was inseparable by reverse phase HPLC on $\mathrm{C}_{18}$ column (MeOH).

Compound 1: $[\alpha]_{\mathrm{D}}^{28}+10.2^{\circ}\left(c=0.45\right.$, pyridine). IR $(\mathrm{KBr}) \mathrm{cm}^{-1}: 3460$, 1630. ${ }^{1} \mathrm{H}-\mathrm{NMR}$ (pyridine- $\left.d_{5}, 300 \mathrm{MHz}\right) \delta: 8.58(1 \mathrm{H}, \mathrm{d}, J=8.7 \mathrm{~Hz}, \mathrm{NH}), 5.09$ $(1 \mathrm{H}, \mathrm{m}, \mathrm{H}-2), 0.87\left(6 \mathrm{H}, \mathrm{t}, J=6.8 \mathrm{~Hz}, \mathrm{CH}_{3}\right) .{ }^{13} \mathrm{C}-\mathrm{NMR}$ (pyridine- $\left.d_{5}, 75 \mathrm{MHz}\right)$ $\delta: 62.1$ (t, C-1), $175.3\left(\mathrm{~s}, \mathrm{C}-1^{\prime}\right), 53.0$ (d, C-2), 73.0 (d, C-2'), 76.8 (d, C-3), $34.8\left(\mathrm{t}, \mathrm{C}-3^{\prime}\right), 72.5(\mathrm{~d}, \mathrm{C}-4), 34.2(\mathrm{t}, \mathrm{C}-5), 30.4-25.9(\mathrm{t}), 14.3(\mathrm{q}$, end methyls). FAB-MS $m / z: 698[\mathrm{M}+\mathrm{H}]^{+}, 382,300,282,264$. Anal. Calcd for $\mathrm{C}_{43} \mathrm{H}_{87} \mathrm{NO}_{5}$ : C, 73.96; H, 12.57; N, 2.01. Found: C, 74.09; H, 12.61; N, 1.97. 
Compound 2: $[\alpha]_{\mathrm{D}}^{28}+12.0^{\circ}(c=0.15$, pyridine $)$. IR $(\mathrm{KBr}) \mathrm{cm}^{-1}: 3500$ 3200, 2940, 1630. ${ }^{1} \mathrm{H}-\mathrm{NMR}$ (pyridine- $\left.d_{5}, 300 \mathrm{MHz}\right) \delta: 8.61(1 \mathrm{H}, \mathrm{d}, J=9 \mathrm{~Hz}$, $\mathrm{NH}), 4.75$ (1H, dd, $J=7,10 \mathrm{~Hz}, \mathrm{H}-1 \mathrm{a}), 4.55(1 \mathrm{H}, \mathrm{dd}, J=4,10 \mathrm{~Hz}, \mathrm{H}-1 \mathrm{~b})$, $5.29(1 \mathrm{H}, \mathrm{m}, \mathrm{H}-2), 4.34(1 \mathrm{H}, \mathrm{dd}, J=5,7 \mathrm{~Hz}, \mathrm{H}-3), 4.20(1 \mathrm{H}, \mathrm{m}, \mathrm{H}-4), 1.90$ (2H, m, H-5), $1.66(2 \mathrm{H}, \mathrm{m}, \mathrm{H}-6), 4.64\left(1 \mathrm{H}, \mathrm{dd}, J=8,4 \mathrm{~Hz}, \mathrm{H}-2^{\prime}\right), 2.88(1 \mathrm{H}$, m, H-3a' $3.05\left(1 \mathrm{H}, \mathrm{m}, \mathrm{H}-3 \mathrm{~b}^{\prime}\right), 5.96\left(1 \mathrm{H}, \mathrm{dt}, J=7.2,18.0 \mathrm{~Hz}, \mathrm{H}-4^{\prime}\right), 5.65$ $\left(1 \mathrm{H}, \mathrm{dt}, J=7.2,17.7 \mathrm{~Hz}, \mathrm{H}-5^{\prime}\right), 2.16\left(2 \mathrm{H}, \mathrm{m}, \mathrm{H}-6^{\prime}\right), 1.29\left(56 \mathrm{H}\right.$, br s, $\left.-\mathrm{CH}_{2}-\right)$, $0.85(6 \mathrm{H}, \mathrm{t}, J=6.9 \mathrm{~Hz}$, end methyls $), 4.94\left(1 \mathrm{H}, \mathrm{d}, J=8 \mathrm{~Hz}, \mathrm{H}-1^{\prime \prime}\right), 4.03(1 \mathrm{H}$, dd, $\left.J=8,9 \mathrm{~Hz}, \mathrm{H}-2^{\prime \prime}\right), 4.18\left(1 \mathrm{H}, \mathrm{m}, \mathrm{H}-3^{\prime \prime}\right), 4.21\left(1 \mathrm{H}, \mathrm{m}, \mathrm{H}-4^{\prime \prime}\right), 3.86(1 \mathrm{H}$, br s, H-5"), 4.31 ( $1 \mathrm{H}$, dd, $\left.J=6,12 \mathrm{~Hz}, \mathrm{H}-6 \mathrm{a}^{\prime \prime}\right), 4.50$ (1H, dd, $\left.J=4,12 \mathrm{~Hz}, \mathrm{H}-6 \mathrm{~b}^{\prime \prime}\right)$. ${ }^{13} \mathrm{C}-\mathrm{NMR}$ (pyridine- $\left.d_{5}, 75 \mathrm{MHz}\right) \delta: 70.5(\mathrm{t}, \mathrm{C}-1), 51.8(\mathrm{~d}, \mathrm{C}-2), 75.8(\mathrm{~d}, \mathrm{C}-$ 3), 72.5 (d, C-4, C-2'), 34.2 (t, C-5), 175.0 (s, C-1'), $33.6\left(\mathrm{t}, \mathrm{C}-3^{\prime}\right), 126.3$ (d, $\left.\mathrm{C}-4^{\prime}\right), 132.4\left(\mathrm{~d}, \mathrm{C}-5^{\prime}\right), 32.2\left(\mathrm{t}, \mathrm{C}-6^{\prime}\right), 30.4-23.0\left(\mathrm{t},-\mathrm{CH}_{2}-\right), 14.3(\mathrm{q}$, end methyls), 105.6 (d, C-1"), 75.2 (d, C-2"), 78.5 (d, C-3"), 71.5 (d, C-4"), 78.6 $\left(\mathrm{d}, \mathrm{C}-5^{\prime \prime}\right), 62.6$ (t, C-6"). FAB-MS m/z: 844 [M+H], 664, 348, 300, 282, 264. Anal. Calcd for $\mathrm{C}_{48} \mathrm{H}_{93} \mathrm{NO}_{10}$ : C, 68.27; H, 11.11; N, 1.66. Found: C, 68.19; $\mathrm{H}, 11.24 ; \mathrm{N}, 1.73$.

Compound 3a-d: Mixture melted at $121-123^{\circ} \mathrm{C}$. IR $(\mathrm{KBr}) \mathrm{cm}^{-1}: 3500$, 1640. ${ }^{1} \mathrm{H}-\mathrm{NMR}$ (pyridine- $\left.d_{5}, 300 \mathrm{MHz}\right) \delta: 8.50(\mathrm{~d}, J=7.4 \mathrm{~Hz}, \mathrm{NH}), 4.49$ (dd, $J=3,11 \mathrm{~Hz}, \mathrm{H}-1 \mathrm{a}), 4.48$ (dd, $J=4.2,11 \mathrm{~Hz}, \mathrm{H}-1 \mathrm{~b}), 5.08$ (m, H-2), 4.39 (dd, $J=5,3 \mathrm{~Hz}, \mathrm{H}-3), 4.28$ (m, H-4), 1.96 (m, H-5), 1.69 (m, H-6), 2.45 (t, $J=7.5 \mathrm{~Hz}, \mathrm{H}-2^{\prime}$ ), 2.22 (m, H-3'), 1.85 (m, H-4'), 1.30 (br s, $-\mathrm{CH}_{2}-$ ), 0.86 (t, $J=3.2 \mathrm{~Hz}$, end methyls). ${ }^{13} \mathrm{C}-\mathrm{NMR}$ (pyridine- $\left.d_{5}, 75 \mathrm{MHz}\right) \delta: 62.2(\mathrm{t}, \mathrm{C}-1)$, 53.8 (d, C-2), 76.7 (d, C-3), 73.1 (d, C-4), 33.9 (t, C-5), 173.4 (s, C-1'), 36.9 $\left(\mathrm{t}, \mathrm{C}-2^{\prime}\right), 32.1\left(\mathrm{t}, \mathrm{C}-3^{\prime}\right), 32.1\left(\mathrm{t}, \mathrm{C}-4^{\prime}\right), 30.3-23.0\left(\mathrm{t},-\mathrm{CH}_{2}-\right), 14.3$ (q, end methyls). FAB-MS $m / z: 654,668,682,696[\mathrm{M}+\mathrm{H}]^{+}$.

Acetylation Dry pyridine $(0.5 \mathrm{ml})$ and $\mathrm{Ac}_{2} \mathrm{O}(1.0 \mathrm{ml})$ were added to compounds 1 (25 mg), 2 (10 mg) and $3(10 \mathrm{mg})$ separately and left overnight. After usual workup and crystallisation in each case yielded 1a, 2a and $\mathbf{3 e}$.

Compound 1a: $\operatorname{mp} 61^{\circ} \mathrm{C},[\alpha]_{\mathrm{D}}^{28}+11.0^{\circ} \quad\left(c=0.1, \mathrm{CHCl}_{3}\right) .{ }^{1} \mathrm{H}-\mathrm{NMR}$ $\left(\mathrm{CDCl}_{3}, 300 \mathrm{MHz}\right) \delta: 6.62(1 \mathrm{H}, \mathrm{d}, J=9 \mathrm{~Hz}, \mathrm{NH}), 4.34(1 \mathrm{H}, \mathrm{dd}, J=6.6$, $11.4 \mathrm{~Hz}, \mathrm{H}-1 \mathrm{a}), 4.02$ (1H, dd, $J=3,11.7 \mathrm{~Hz}, \mathrm{H}-1 \mathrm{~b}), 4.44$ (1H, m, H-2), 5.10 $\left(2 \mathrm{H}, \mathrm{dd}, J=6.9,10.8 \mathrm{~Hz}, \mathrm{H}-3, \mathrm{H}-2^{\prime}\right), 4.95$ (1H, m, H-4), 1.69 (4H, m, H-5, H-4'), $1.84\left(2 \mathrm{H}, \mathrm{m}, \mathrm{H}-3^{\prime}\right), 1.25\left(64 \mathrm{H}\right.$, br s, $\left.-\mathrm{CH}_{2}-\right), 0.85(6 \mathrm{H}, \mathrm{t}, J=6.0 \mathrm{~Hz}$, end methyls), $2.03-2.18\left(12 \mathrm{H}, \mathrm{s}, 4-\mathrm{COCH}_{3}\right) .{ }^{13} \mathrm{C}-\mathrm{NMR}\left(\mathrm{CDCl}_{3}, 75 \mathrm{MHz}\right)$ $\delta$ : $62.4(\mathrm{t}, \mathrm{C}-1), 47.8$ (d, C-2), 74.0 (d, C-3), 72.3 (d, C-4), 31.8 (t, C-5), $171.3\left(\mathrm{~s}, \mathrm{C}-1^{\prime}\right), 72.7\left(\mathrm{~d}, \mathrm{C}-2^{\prime}\right), 31.9\left(\mathrm{t}, \mathrm{C}-3^{\prime}\right), 31.8\left(\mathrm{t}, \mathrm{C}-4^{\prime}\right), 29.7-22.7$ $\left(\mathrm{t},-\mathrm{CH}_{2}-\right), 14.1\left(\mathrm{q},-\mathrm{CH}_{3}\right), 20.6-21.0\left(\mathrm{~s},-\mathrm{COCH}_{3}\right), 170.0-170.9$ (s, $\left.\mathrm{COCH}_{3}\right)$. FAB-MS $m / z: 866[\mathrm{M}+\mathrm{H}]^{+}$.

Compound 2a: mp $57^{\circ} \mathrm{C}$. ${ }^{1} \mathrm{H}-\mathrm{NMR}\left(\mathrm{CDCl}_{3}, 300 \mathrm{MHz}\right) \delta: 6.80(1 \mathrm{H}, \mathrm{d}$, $J=9.0 \mathrm{~Hz}, \mathrm{NH}), 4.28(1 \mathrm{H}, \mathrm{m}, \mathrm{H}-2), 1.99-2.24\left(21 \mathrm{H}, \mathrm{s}, 7-\mathrm{COCH}_{3}\right), 1.25$ (br s, $\left.-\mathrm{CH}_{2}-\right), 0.86(6 \mathrm{H}, \mathrm{t}, J=6.8 \mathrm{~Hz}$, end methyls).

Compound 3e: $\mathrm{mp} 65^{\circ} \mathrm{C}$. ${ }^{1} \mathrm{H}-\mathrm{NMR}\left(\mathrm{CDCl}_{3}, 300 \mathrm{MHz}\right) \delta: 6.62(\mathrm{~d}, J=$ $9 \mathrm{~Hz}, \mathrm{NH}), 1.90-2.01\left(\mathrm{~s},-\mathrm{COCH}_{3}\right), 1.25\left(\mathrm{br} \mathrm{s},-\mathrm{CH}_{2}-\right), 0.85(\mathrm{t}, J=5.7 \mathrm{~Hz}$, end methyls). FAB-MS $m / z: 766,780,794,808\left[\mathrm{M}+\mathrm{H}^{+}{ }^{+}\right.$.

Methanolysis Compounds $\mathbf{1}$ and $\mathbf{3}(10 \mathrm{mg})$ were separately treated with $3 \mathrm{ml}$ of $1 \mathrm{~N} \mathrm{HCl}$ in methanol at $90^{\circ} \mathrm{C}$ for $15 \mathrm{~h}$ with magnetic stirring. The fatty acid methyl ester so obtained in each case was extracted with $n$-hexane and analyzed by GC-MS. In each case a single compound was obtained. Methyl ester from Compound 1: $[\alpha]_{\mathrm{D}}^{28}-2.6^{\circ}\left(c=0.05, \mathrm{CHCl}_{3}\right) .{ }^{1} \mathrm{H}-\mathrm{NMR}$ $\left(\mathrm{CDCl}_{3}, 300 \mathrm{MHz}\right) \delta: 5.13(1 \mathrm{H}, \mathrm{dd}, J=6.9,3.9 \mathrm{~Hz}, \mathrm{H}-2), 3.52(3 \mathrm{H}, \mathrm{s}$, $\left.\mathrm{OCH}_{3}\right), 1.84(2 \mathrm{H}, \mathrm{m}, \mathrm{H}-3), 1.68(2 \mathrm{H}, \mathrm{m}, \mathrm{H}-4), 1.25\left(42 \mathrm{H}\right.$, br s, $\left.-\mathrm{CH}_{2}-\right), 0.88$ $\left(3 \mathrm{H}, \mathrm{t}, \mathrm{CH}_{3}\right)$. GC-MS m/z: $412[\mathrm{M}]^{+}$. Methyl ester from Compound 3: ${ }^{1} \mathrm{H}-$ NMR $\left(\mathrm{CDCl}_{3}, 300 \mathrm{MHz}\right) \delta: 2.28(2 \mathrm{H}, \mathrm{t}, J=7.2 \mathrm{~Hz}, \mathrm{H}-2), 3.57(3 \mathrm{H}, \mathrm{s}$, $\left.\mathrm{OCH}_{3}\right), 1.84(2 \mathrm{H}, \mathrm{m}, \mathrm{H}-3), 1.30\left(36 \mathrm{H}\right.$, br s, $\left.-\mathrm{CH}_{2}-\right), 0.85(3 \mathrm{H}, \mathrm{t}, J=7.5 \mathrm{~Hz}$, $\mathrm{CH}_{3}$ ). GC-MS m/z: $354[\mathrm{M}]^{+}$. The $\mathrm{MeOH} / \mathrm{H}_{2} \mathrm{O}$ phase was evaporated and the residue was acetylated. Purification by filtration over a Sephadex LH20 column $\left(\mathrm{CH}_{2} \mathrm{Cl}_{2} / \mathrm{MeOH}, 1: 1\right)$ gave the acetylated sphingamines. The sphingamines were subjected to GC-MS in each case. Acetylated sphingamines from Compound 1: $[\alpha]_{\mathrm{D}}^{28}+24.2^{\circ}\left(c=0.05, \mathrm{CHCl}_{3}\right) .{ }^{1} \mathrm{H}-\mathrm{NMR}$ $\left(\mathrm{CDCl}_{3}, 300 \mathrm{MHz}\right) \delta: 6.61(1 \mathrm{H}, \mathrm{d}, J=9 \mathrm{~Hz}, \mathrm{NH}), 5.10(1 \mathrm{H}, \mathrm{dd}, J=7,4 \mathrm{~Hz}$, H-3), 4.96 (1H, m, H-4), 4.44 (1H, m, H-2), 4.35 (1H, dd, J=6.2, $10.4 \mathrm{~Hz}$, $\mathrm{H}-1 \mathrm{a}), 4.03(1 \mathrm{H}, \mathrm{dd}, J=4.1,10.6 \mathrm{~Hz}, \mathrm{H}-1 \mathrm{~b}), 2.18-2.03\left(12 \mathrm{H}, \mathrm{s}, 4-\mathrm{COCH}_{3}\right)$, $1.68(2 \mathrm{H}, \mathrm{m}, \mathrm{H}-5), 1.25\left(24 \mathrm{H}\right.$, br s, $\left.-\mathrm{CH}_{2}-\right), 0.88\left(3 \mathrm{H}, \mathrm{t}, J=5.8 \mathrm{~Hz}, \mathrm{CH}_{3}\right)$. GC-MS $m / z$ : $485\left[\mathrm{M}^{+}\right.$. Acetylated sphingamines from Compound $3 \mathbf{a}-\mathbf{d}$ :
${ }^{1} \mathrm{H}-\mathrm{NMR}\left(\mathrm{CDCl}_{3}, 300 \mathrm{MHz}\right) \delta: 6.60(\mathrm{~d}, J=9 \mathrm{~Hz}, \mathrm{NH}), 5.10(\mathrm{dd}, J=4.5$, $7.8 \mathrm{~Hz}, \mathrm{H}-3$ ), 4.93 (dd, J=3.7, $9.8 \mathrm{~Hz}, \mathrm{H}-1 \mathrm{~b}), 4.63$ (m, H-4), 4.44 (m, H-2), 4.39 (dd, $J=4.6,9.6 \mathrm{~Hz}, \mathrm{H}-1 \mathrm{a}), 2.18-2.03\left(\mathrm{~s}, 4-\mathrm{COCH}_{3}\right), 1.84$ (m, H-4), 1.65 (m, H-5), 1.25 (br s, $-\mathrm{CH}_{2}-$ ), 0.85 (t, $J=6.2 \mathrm{~Hz}, \mathrm{CH}_{3}$ ). GC-MS m/z: 499 $(23 \%), 513(26 \%), 527(23 \%), 541(28 \%)[\mathrm{M}]^{+}$.

The process was repeated with compound $2(8 \mathrm{mg})$. The methyl ester so obtained was analysed by GC-MS which showed single component with $m / z: 396[\mathrm{M}]^{+} .[\alpha]_{\mathrm{D}}^{28}-2.4^{\circ}\left(c=0.05, \mathrm{CHCl}_{3}\right),{ }^{1} \mathrm{H}-\mathrm{NMR}\left(\mathrm{CDCl}_{3}, 300 \mathrm{MHz}\right)$ $\delta: 5.54(1 \mathrm{H}, \mathrm{dt}, J=17.4,7.2 \mathrm{~Hz}, \mathrm{H}-4), 5.34(1 \mathrm{H}, \mathrm{dt}, J=17.7,7.2 \mathrm{~Hz}, \mathrm{H}-5)$, $4.73(1 \mathrm{H}, \mathrm{dd}, J=8,4 \mathrm{~Hz}, \mathrm{H}-2), 3.61\left(3 \mathrm{H}, \mathrm{s}, \mathrm{OCH}_{3}\right), 2.18(1 \mathrm{H}, \mathrm{m}, \mathrm{H}-3 \mathrm{a}), 1.98$ $(1 \mathrm{H}, \mathrm{m}, \mathrm{H}-3 \mathrm{~b}), 1.25\left(34 \mathrm{H}\right.$, br s, $\left.-\mathrm{CH}_{2}-\right), 0.86(3 \mathrm{H}, \mathrm{t}, J=8.4 \mathrm{~Hz}$, end methyl). The aqueous methanolic layer of the hydrolysate was evaporated to dryness and the residue was separated by TLC as sphingosine base and methylated sugar. The base was acetylated and subjected to GC-MS $m / z: 485[\mathrm{M}]^{+}$, $[\alpha]_{\mathrm{D}}^{28}+26.7^{\circ}\left(c=0.05, \mathrm{CHCl}_{3}\right),{ }^{1} \mathrm{H}-\mathrm{NMR}\left(\mathrm{CDCl}_{3}, 300 \mathrm{MHz}\right) \delta: 6.81(1 \mathrm{H}, \mathrm{d}$, $J=9 \mathrm{~Hz}, \mathrm{NH}), 5.30(1 \mathrm{H}, \mathrm{dd}, J=4.1,7.3 \mathrm{~Hz}, \mathrm{H}-3), 4.95$ (1H, m, H-4), 4.43 (1H, dd, $J=10.2,3.9 \mathrm{~Hz}, \mathrm{H}-1 \mathrm{~b}), 4.28(1 \mathrm{H}, \mathrm{m}, \mathrm{H}-2), 4.12(1 \mathrm{H}, \mathrm{dd}, J=10.2$, $4.3 \mathrm{~Hz}, \mathrm{H}-1 \mathrm{a}), 1.78$ (2H, m, H-5), 1.56 (2H, m, H-6), 2.02-2.12 (12H, s, 4$\left.\mathrm{COCH}_{3}\right), 1.25\left(22 \mathrm{H}\right.$, br s, $\left.-\mathrm{CH}_{2}-\right), 0.86(3 \mathrm{H}, \mathrm{t}, J=8.4 \mathrm{~Hz}$, end methyl). The presence of methyl- $\beta$-D-glucopyranose was established by comparison of NMR data with those reported in the literature and with an authentic sample $[\alpha]_{\mathrm{D}}^{28}+74^{\circ}(c=0.1, \mathrm{MeOH}), R f 0.46\left(\mathrm{EtOAc} / \mathrm{MeOH} / \mathrm{H}_{2} \mathrm{O}, 5: 2: 0.5\right)$. Authentic sample $[\alpha]_{\mathrm{D}}^{28}+77^{\circ}(c=0.1, \mathrm{MeOH})$, Rf 0.46 .

Acknowledgements The authors thank Dr. P. A. Thomas, CMFRI, Trivandrum for identification of the organism, The University Grants Commission, Dept. of Ocean Development, AICTE, New Delhi for financial support. Thanks are due to RSIC, CDRI, Lucknow for spectral data.

\section{References and Notes}

1) Present address: Bhargavi, 9-40-7, Pithapuram Colony, Visakhapatnam, India.

2) Karlsson K. A., "Biological Membranes," Vol. 4, ed. by Chapman D., Academic Press, London, 1982, p. 1.

3) John W. B., Brent R. C., Murray H. G. M., Peter T. N., Michèle R. P. Nat. Prod. Rep., 20, 1-48 (2003).

4) Faulkner D. J., Nat. Prod. Rep., 19, 1-48 (2002), and earlier annual reports.

5) Jin W., Rinehart K. L., Jares-Erijman E. A., J. Org. Chem., 59, 144147 (1994).

6) Chebaane K., Guyot M., Tetrahedron Lett., 27, 1495-1496 (1986).

7) Bheemasankara Rao Ch., Satyanarayana Ch., Indian J. Chem., 33B, 97-98 (1994).

8) Gaver R. C., Sweeley C. C., J. Am. Oil. Chem. Soc., 42, 294-298 (1965).

9) Sugiyama S., Honda M., Komori T., Liebigs Ann. Chem., 1988, 619625 (1988).

10) Moon D.-C., Hwang K.-H., Choi K.-R., Lee Y.-M., Kim J.-H., Kim J.G., Park Y.-H., Kim M.-R., Zee O.-P., Anal. Sci. Technol., 7, 547—554 (1994).

11) Yaoita Y., Ishizuka T., Kakuda R., Machida K., Kikuchi M., Chem. Pharm. Bull., 48, 1356-1358 (2000).

12) Bock K., Pederson C., "Advances in Carbohydrate Chemistry and Biochemistry," Vol. 41, eds.by Jipson R. S., Horfon D., Academic Press, New York, 1983, pp. 27-46.

13) Silverstein R. M., Webster F. X., "Spectrometric Identification of Organic Compounds," 6th ed., John Wiley \& Sons, Inc., New York, 1998.

14) Fusetani N., Yasumoto K., Matsunaga S., Hirota H., Tetrahedron Lett., 30, 6891-6894 (1989).

15) Deng S.-Z., Tian C.-L., Xiao D.-J., Wu H.-M., Chin. J. Chem., 19, 362-364 (2001).

16) Meng Y., Su J., Zeng L., Zhongshan Daxue Xuebao, Ziran Kexueban, 35, 69-72 (1996).

17) Wild R., Schimdt R. R., Liebigs Ann. Chem., 1995, 755-764 (1995). 